\title{
Multifrequency simulations of the EPR spectra of lipid spin labels in membranes
}

\author{
V.A. Livshits ${ }^{\mathrm{b}}$, Dieter Kurad ${ }^{\mathrm{a}}$, D. Marsh ${ }^{\mathrm{a}, *}$ \\ ${ }^{a}$ Max-Planck-Institut für biophysikalische Chemie, Abt. Spektroskopie, 37077 Göttingen, Germany \\ ${ }^{\mathrm{b}}$ Centre of Photochemistry, Russian Academy of Sciences, 117427 Moscow, Russian Federation
}

Received 3 November 2005; revised 10 January 2006

Available online 31 January 2006

\begin{abstract}
Simulations are performed of 34- and 9-GHz EPR spectra, together with 94-GHz EPR spectra, from phospholipid probes spin-labelled at the $\mathrm{C} 4-\mathrm{C} 14$ positions of the sn-2 chain, in liquid-ordered and gel-phase membranes of dimyristoyl phosphatidylcholine with high and low cholesterol contents. The multifrequency simulation strategy involves: (i) obtaining partially averaged spin-Hamiltonian tensors from fast-motional simulations of the 94-GHz spectra; (ii) performing slow-motional simulations of the 34- and 9-GHz spectra by using these pre-averaged tensors with the stochastic Liouville formalism; (iii) constructing, by simulation, slow-motional calibrations for the differences, $\Delta A_{z z}^{q x}$ and $\Delta g_{z z}^{q x}$, in effective $A_{z z}$-hyperfine splittings and $g_{z z}$-values between 34- (or 94-GHz) and 9-GHz spectra; (iv) using such calibrations for $\Delta A_{z z}^{q x}$ and $\Delta g_{z z}^{q x}$ and dynamic parameters from stage (ii) as a guide to adjust the extent of pre-averaging of the spin-Hamiltonian tensors; and (v) repeating the 34- and 9-GHz simulations of stage (ii). By using this scheme it is possible to obtain consistent values of the rotational diffusion coefficients, $D_{R \perp}$ and $D_{R / /}$, and the long-axis order parameter, $S_{z z}$, that characterize the slow axial motion of the lipid chains, from spectra at both 34 and $9 \mathrm{GHz}$. Inclusion of spectra at $34 \mathrm{GHz}$ greatly improves precision in determining the $D_{R / /}$ element of the slow diffusion tensor in these systems.
\end{abstract}

(C) 2006 Elsevier Inc. All rights reserved.

Keywords: High frequency; EPR; Lipid spin label; Stochastic Liouville; Brownian diffusion

\section{Introduction}

The dynamics of lipid molecules in biological membranes are known to be composite and manifested on different time scales. In addition to relatively slow overall reorientations in the locally anisotropic environment, there are also rapid segmental chain motions that arise from internal rotations about individual $\mathrm{C}-\mathrm{C}$ bonds, viz., trans-gauche isomerism (see, e.g., [1]). EPR spectroscopy of lipid spin labels at variable microwave frequency is thus a promising approach for studying lipid dynamics, because the spin-label EPR spectra are sensitive to the rapid and slow motions to different extents depending on the operating frequency. Earlier, spectral simulations that use simpli-

\footnotetext{
* Corresponding author. Fax: +49 5512011501

E-mail address: dmarsh@gwdg.de (D. Marsh).
}

fied but physically reasonable models of lipid motion proved to be relatively successful in interpreting the highfield (250- and 94-GHz) and low-field (9-GHz) EPR spectra of lipid spin labels in membranes [2,3]. Combined study at high-field and low-field allows one to estimate dynamic parameters both of the fast internal modes and of the slower overall motion [4]. However, it is expected that a deeper understanding and critical testing of the models for lipid motion can be achieved by using additional microwave frequencies, particularly $Q$-band ( $34 \mathrm{GHz}$ ) which is intermediate between the $W$ - and $X$-bands (94 and 9; GHz, respectively). Most importantly, measurements at $34 \mathrm{GHz}$ are able to define the axial component of the slow rotation, to which measurements at $9 \mathrm{GHz}$ are rather insensitive. The purpose of the present work, therefore, is to explore the potential of combined EPR measurements, at the above three microwave frequencies, for studying the dynamics of phospholipid spin labels in membranes. 
An additional approach to determining the dynamic parameters that characterize the lipid motion is to develop calibrations for motion-sensitive parameters of the EPR spectra at different microwave frequencies, in terms of a given dynamic model. These calibrations then can also be used for testing the motional model and consistency of the parameters derived from it. Hyde and Rao [5] proposed a method for determining rotational correlation times and rigid-limit hyperfine splittings $\left(A_{z z}\right)$ by measuring the partially averaged $\left\langle A_{z z}\right\rangle$-values in $X$ - and $Q$-band spin-label spectra. These authors showed by spectral simulation that the difference: $\Delta A_{z z}^{q x}=\left\langle A_{z z}(Q)\right\rangle-\left\langle A_{z z}(X)\right\rangle$, is not sensitive to the input magnetic parameters and depends only on the rotational correlation time $\left(\tau_{R}\right)$, thus allowing determination of $\tau_{R}$ from calibrations, without knowledge of the hyperfine tensor element $A_{z z}$. The apparent rigid-limit values of $A_{z z}$ are of intrinsic interest themselves, because these are determined by the amplitude of any fast motion that might be present. If this motion is very fast, it gives rise to an averaged spin-Hamiltonian. According to Hyde and Rao [5], the values of $A_{z z}$, and hence the amplitudes of fast motion, can be determined from theoretical plots of $A_{z z}-\left\langle A_{z z}\right\rangle$ versus $\tau_{R}$ that are calculated for 9 or $34 \mathrm{GHz}$.

The above multifrequency approach was developed for isotropic rotational diffusion in an isotropic medium. In lipid membranes, the situation is more complex because the lipid motion is anisotropic and occurs in a locally anisotropic environment. Therefore, the overall motion of the lipid molecules, described in terms of the Brownian diffusion model, is characterized by at least three dynamic parameters: rotational diffusion coefficients for off-axial and axial motion $\left(D_{R \perp}, D_{R / /}\right)$, and an order parameter $\left(S_{z z}\right)$ that is determined by the self-consistent local orientation potential [6]. Here, in addition to simulations, we also construct multifrequency calibration parameters $\left(\Delta A_{z z}^{q x}\right.$ and $\left.\Delta g_{z z}^{q x}\right)$ for this slow anisotropic diffusion and show that they are a valuable aid to achieving consistent simulations at 9 and $34 \mathrm{GHz}$.

\section{Materials and methods}

\subsection{Materials}

Dimyristoyl phosphatidylcholine (DMPC) was obtained from Avanti Polar Lipids (Alabaster, AL) and cholesterol from Merck (Darmstadt, Germany). Spin-labelled stearic acid positional isomers ( $n$-(4,4-dimethyloxazolidine- $N$-oxyl)stearic acid) were synthesised as described in [7]. The corresponding phosphatidylcholines spin-labelled in the $s n$ - 2 chain (1-acyl-2-[n-(4,4-dimethyloxazolidine- $N$-oxyl)] stearoyl-sn-glycero-3-phosphocholine; $n$-PCSL) were synthesised by acylating lysophosphatidylcholine with the corresponding spin-labelled stearic acid, as described in [8].

\subsection{Sample preparation}

DMPC, the required mole percentage of cholesterol and $0.5 \mathrm{~mol} \% n$-PCSL spin-label were codissolved in dichloromethane. The solvent was evaporated under a nitrogen gas stream, and the samples were dried under vacuum overnight. The dried lipid mixtures were then hydrated with an excess of buffer $(100 \mathrm{mM} \mathrm{KCl}, 10 \mathrm{mM}$ Tris, and $1 \mathrm{mM}$ EDTA) at $\mathrm{pH}$ 7.5. The resulting lipid dispersions were introduced into $0.5 \mathrm{~mm}$ ID glass capillaries, and then concentrated by centrifugation and the excess aqueous supernatant was removed. The capillaries were flame sealed.

\subsection{EPR spectroscopy}

EPR spectra were recorded at $34 \mathrm{GHz}$ ( $Q$-band) on a Bruker EMX spectrometer with ER-035 QRD microwave bridge and a cylindrical cavity resonator. The whole resonator assembly was thermostatted with nitrogen gas-flow. Microwave frequency was measured with a Hewlett-Packard 5345/55A frequency counter and the static magnetic field with a Bruker ER 035M NMR gaussmeter.

Data recorded at 94 and $9 \mathrm{GHz}$ are taken from previous studies [3,9], and were recorded on Bruker Elexys E680 and EMX spectrometers, respectively.

\subsection{Spectral simulations}

Simulation of 34- and 9-GHz spectra from spin labels undergoing overall slow rotational motion, in addition to a rapid segmental component, is performed by means of the stochastic Liouville equation with a spin-Hamiltonian $\langle\mathscr{H}(\Omega)\rangle$ that contains the $g$ - and $A$-tensors that are averaged (partially) by the rapid motion [3]. This type of timescale separation is possible when the fast and slow motions differ very greatly in their rates [10]. A more general approach to this problem is afforded by the "slowly relaxing local structure" model $[2,11]$.

The slow motion is characterized by an axial diffusion tensor, $\mathbf{D}_{\mathbf{R}}=\left(D_{R \perp}, D_{R \perp}, D_{R / /}\right)$, and by an orientational pseudopotential that is expanded in terms of Wigner rotation matrices, $\mathscr{D}_{K, M}^{L}(\Omega)[6]$ :

$$
U(\Omega)=\varepsilon_{20} \mathscr{D}_{0,0}^{2}(\Omega)+\varepsilon_{22}\left[\mathscr{D}_{2,0}^{2}(\Omega)+\mathscr{D}_{-2,0}^{2}(\Omega)\right],
$$

where the $\varepsilon_{i}$ represent the strength of the orienting potential. The principal order parameter of the slow motion is then given by

$S_{z z}=\left\langle\mathscr{D}_{0,0}^{2}\right\rangle=\frac{\int \mathscr{D}_{0,0}^{2}(\Omega) \exp [-U(\Omega) / k T] \mathrm{d} \Omega}{\int \exp [-U(\Omega) / k T] \cdot \mathrm{d} \Omega}$,

where averaging is performed over all angular orientations, $\Omega$. Only the $\varepsilon_{20}$ term in Eq. (1) contributes to this element of the order tensor.

The partially averaged $g$ - and $A$-tensors needed for the 34- and 9-GHz simulations are obtained from corresponding $94-\mathrm{GHz}$ spin-label spectra [3]. Simulations are made 
using the software described in [12] with extensions for least-squares fitting by Budil et al. [13]. Because the membrane samples are random dispersions, the simulated spectra are weighted by $\sin \theta_{N}$ and integrated over the angle, $\theta_{N}$, that the membrane normal makes to the static magnetic field. Goodness of fit to the experimental 34- and $9-\mathrm{GHz}$ first-derivative EPR spectra is given in terms of the reduced $\chi^{2}$ reported by the non-linear least-squares fitting routine described in [13].

\section{Results and discussion}

\subsection{Simulation of 34-GHz spectra: $\mathrm{DMPC}+40 \mathrm{~mol} \%$ cholesterol}

Fig. 1 (solid lines) shows experimental 34-GHz EPR spectra of different $n$-PCSL spin labels in the liquid-ordered $\left(\mathrm{L}_{\mathrm{o}}\right)$ phase of DMPC bilayer membranes containing 40 $\mathrm{mol} \%$ cholesterol, at $30^{\circ} \mathrm{C}$. Simulations of the spectra for different chain positions, $n$, of the spin-label are given by the dotted lines in Fig. 1. The partially averaged spin-Hamiltonian tensor components that are used to calculate the simulated spectra are those obtained from our previous 94-GHz measurements [3].

Fitting to the experimental spectra was performed in progressive steps. First, the dynamic parameters $D_{R \perp}$, $D_{R / /}$, and $S_{z z}$ were fixed at the values obtained previously from simulations at $9 \mathrm{GHz}$ [3]. These values of $D_{R \perp}(\mathrm{X})$, $D_{R / /}(X)$, and $S_{z z}(X)$ are given in Table 1, along with opti-

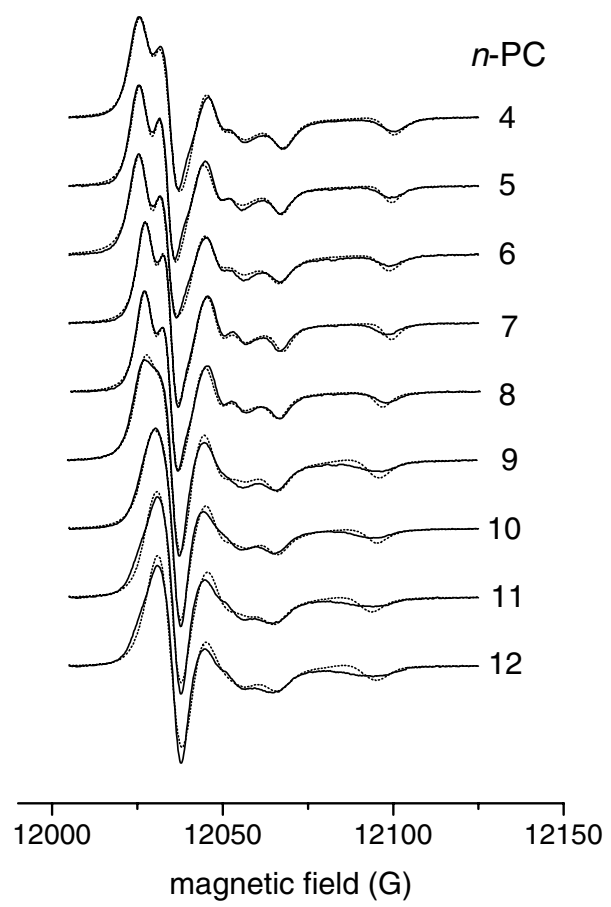

Fig. 1. Experimental (solid lines) and simulated (dotted lines) $34-\mathrm{GHz}$ EPR spectra of $n$-PCSL spin labels in membranes of DMPC $+40 \mathrm{~mol} \%$ cholesterol at $30^{\circ} \mathrm{C}$. The position, $n$, of chain spin-labelling is indicated on the figure. Partially averaged spin-Hamiltonian tensors were obtained from simulation of corresponding $94-\mathrm{GHz}$ spectra. Dynamic parameters used in the simulations are given in Table 1. mized values obtained at various stages of fitting to the $34-\mathrm{GHz}$ spectra. Simulations of the $34-\mathrm{GHz}$ spectra with the initial 9-GHz parameters were mostly not satisfactory. Next, only $D_{R / /}$ was allowed to change, because this parameter cannot be determined precisely from $9-\mathrm{GHz}$ simulations. Varying $D_{R / /}$ resulted, as a rule, in substantially improved fits. Finally, two (either $D_{R / /}$ and $S_{z z}$, or $D_{R \perp}$ and $\left.D_{R / /}\right)$, or all three parameters $\left(D_{R \perp}, D_{R / /}\right.$ and $\left.S_{z z}\right)$, were released in the least-squares optimization procedure. The resulting best simulations are shown in Fig. 1, and the parameters $D_{R \perp}, D_{R / /}$, and $S_{z z}$ obtained from these simulations are given in Table 1, together with the corresponding values of $R \chi^{2}$ for the fit.

As seen from Fig. 1, the agreement between experimental and simulated EPR spectra is, in general, satisfactory. However, because of a general property of the least-squares algorithm, the more intense low-field features are fitted better than the less intense line positions in the central and high-field $z$-regions. Moreover, the fits become worse for larger values of $n$. It is important to note that the values of $D_{R \perp}, D_{R / /}$, and $S_{z z}$ that are obtained from the $34-\mathrm{GHz}$ simulations are rather close (but not identical) to those from the combined 9- and 94-GHz simulations (see Table 1). Differences are found mostly in the values of $D_{R / /}$, which are smaller than for the 9-GHz simulations and vary less with chain position. Determinations of $D_{R / /}$ are far more reliable at $34 \mathrm{GHz}$ than at $9 \mathrm{GHz}$, because the nonaxial Zeeman anisotropy (determined by $g_{x x}-\mathrm{g}_{y y}$ ) is 3-4 times larger at $34 \mathrm{GHz}$ than at $9 \mathrm{GHz}$.

\subsection{Multifrequency hyperfine calibration of dynamic parameters}

As a next step we attempt, following Hyde and Rao [5], to determine the dynamic parameters from spectral calibrations by using the differences in partially averaged hyperfine splittings, $\Delta A_{z z}^{q x}=\left\langle A_{z z}(Q)\right\rangle-\left\langle A_{z z}(X)\right\rangle$ and $\Delta A_{z z}^{w x}=$ $\left\langle A_{z z}(W)\right\rangle-\left\langle A_{z z}(X)\right\rangle$, at the different microwave frequencies, as motionally sensitive spectral parameters. For anisotropic rotation in an orientational potential, these parameters depend on both $D_{R \perp}$ and $S_{z z}$. Calibrations of $\Delta A_{z z}^{w x}$ and $\Delta A_{z z}^{q x}$ versus $D_{R \perp}$ for different values of $S_{z z}$ were calculated by using the stochastic Liouville formalism [13]. Results of these calculations are presented in Fig. 2. The effective values of $\left\langle A_{z z}(W)\right\rangle$ and $\left\langle A_{z z}(X)\right\rangle$ are determined as half the separation between the $m_{I}=+1$ and $m_{I}=-1$ hyperfine components in the $z$-region of the spectrum. That of $\left\langle A_{z z}(Q)\right\rangle$ is determined, correspondingly, as the distance between the $m_{I}=0$ and $m_{I}=-1$ hyperfine components in the $z$-region. It is seen from Fig. 2 that, for low $D_{R \perp}\left(\log D_{R \perp} \leqslant 7.1\right)$, both $\Delta A_{z z}^{w x}$ and $\Delta A_{z z}^{q x}$ are small and are only weakly dependent on $D_{R \perp}$ and $S_{z z}$. Also, the dependences on $D_{R \perp}$ become less sensitive with increasing $S_{z z}$, even for large $D_{R \perp}$.

Experimental values of $\Delta A_{z z}^{w x}$ and $\Delta A_{z z}^{q x}$ that are measured from the EPR spectra of different $n$-PCSL spin labels in membranes of DMPC $+40 \mathrm{~mol} \%$ cholesterol at $30^{\circ} \mathrm{C}$ are given by the solid symbols in Fig. 3. Estimates of $D_{R \perp}$ 
Table 1

Dynamic parameters, $D_{R \perp}(Q), D_{R / /}(Q)$, and $S_{z z}(Q)$, characterising slow overall motion of $n$-PCSL spin labels in membranes of DMPC +40 mol\% cholesterol at $30^{\circ} \mathrm{C}$, obtained from simulations of $34-\mathrm{GHz}$ EPR spectra ${ }^{\mathrm{a}}$

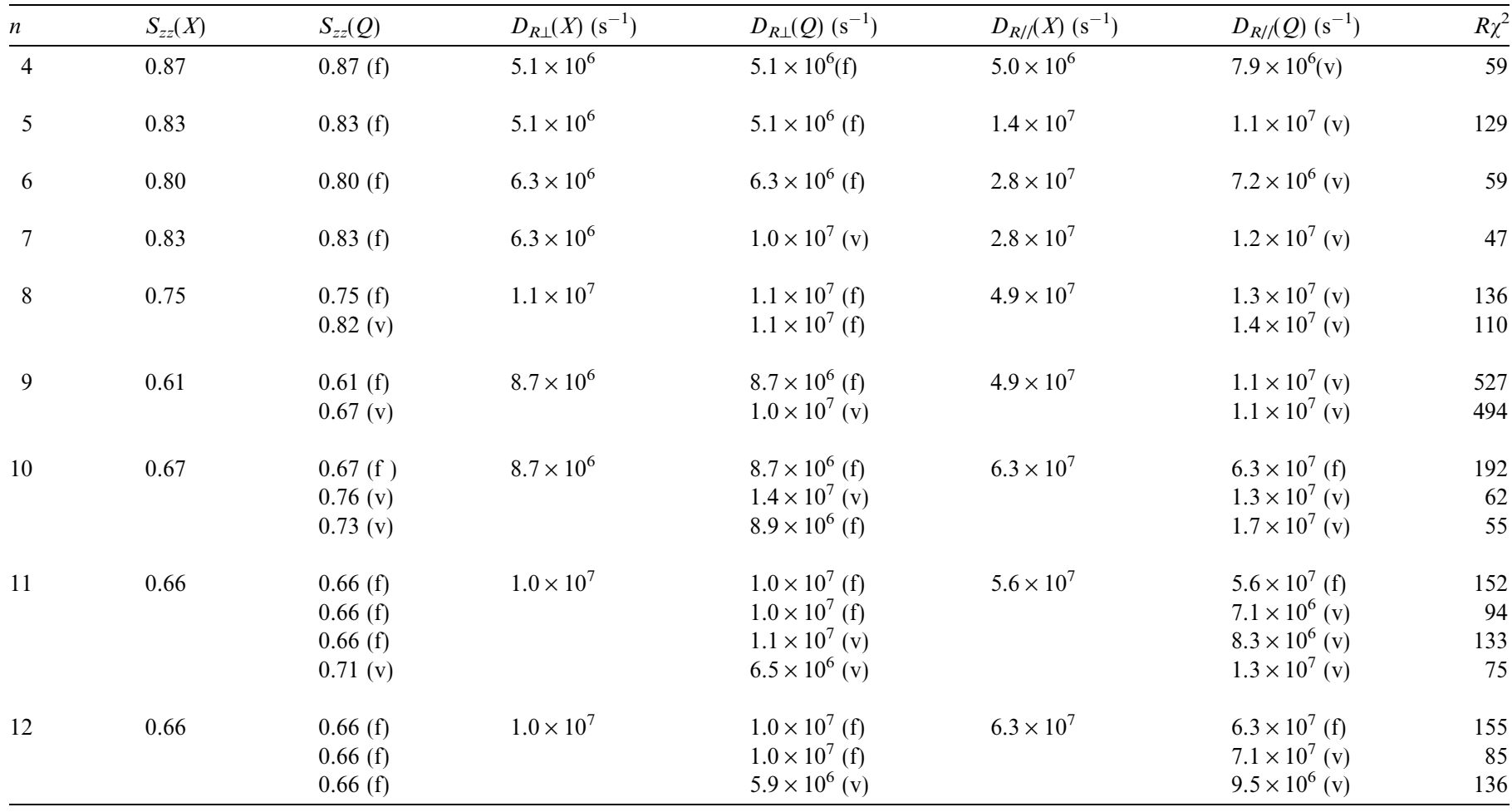

${ }^{\text {a } C o r r e s p o n d i n g ~ i n i t i a l ~ p a r a m e t e r s ~ o b t a i n e d ~ f r o m ~ s i m u l a t i o n ~ o f ~} 9-\mathrm{GHz}$ spectra [3] are given by $D_{R \perp}(X), D_{R / /}(X)$, and $S_{z z}(X)$. Parameters that were maintained fixed during the $34-\mathrm{GHz}$ simulations are indicated by (f) and those that were varied by (v).

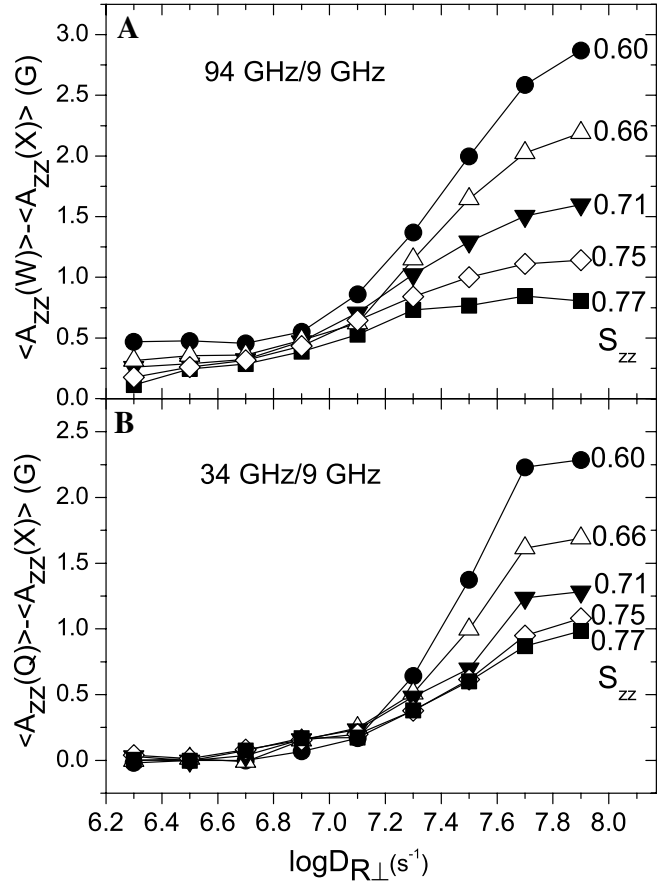

Fig. 2. Dependence of the simulated difference in effective partially averaged hyperfine splittings at $9 \mathrm{GHz}(X), 34 \mathrm{GHz}(Q)$, and $94 \mathrm{GHz}(W)$ : (A) $\Delta A_{z z}^{w x}=\left\langle A_{z z}(W)\right\rangle-\left\langle A_{z z}(X)\right\rangle$, and (B) $\Delta A_{z z}^{q x}=\left\langle A_{z z}(Q)\right\rangle-\left\langle A_{z z}(X)\right\rangle$, on rotational diffusion coefficient $\left(D_{R \perp}\right)$ for different values of the order parameter $\left(S_{z z}\right)$. Spectral simulations are performed with the stochastic Liouville equation. and $S_{z z}$ for 4-PCSL give a range from $2.2 \times 10^{7} \mathrm{~s}^{-1}$ and 0.60 to $5.6 \times 10^{7} \mathrm{~s}^{-1}$ and 0.77 , respectively, derived from measurement of $\Delta A_{z z}^{q x}$, and from $2.2 \times 10^{7} \mathrm{~s}^{-1}$ and 0.60 to $7.9 \times 10^{7} \mathrm{~s}^{-1}$ and 0.75 , respectively, derived from $\Delta A_{z z}^{w x}$. For $n \leqslant 7$, the ranges of $D_{R \perp}$ and $S_{z z}$ remain approximately the same, although the apparent decrease in $\Delta A_{z z}^{q x}$ with increasing $n$ probably lies within the experimental uncertainty. Comparison with the data in Table 1 shows that, for these spin labels, the above ranges correspond to higher rotational diffusion coefficients and/or lower order parameters than those determined from simulation of the experimental spectra. A similar situation holds for the other $n$ PCSL positional isomers.

Clearly, the multifrequency $\Delta A_{z z}$ parameters alone are insufficient criteria for determining the dynamic parameters, in the case of complex, multicomponent, anisotropic motions. As seen from Fig. 2, $\Delta A_{z z}^{w x}$ and $\Delta A_{z z}^{q x}$ depend similarly on $D_{R \perp}$ for different values of $S_{z z}$, which makes it difficult to determine the two separately. Therefore, we choose to use an additional spectral parameter that is based on the partially averaged $g_{z z}$-values.

\subsection{Multifrequency g-value calibration of dynamic parameters}

The partially averaged values of $g_{z z}$, like those of $A_{z z}$, are sensitive only to the off-axis motion. From simulated 


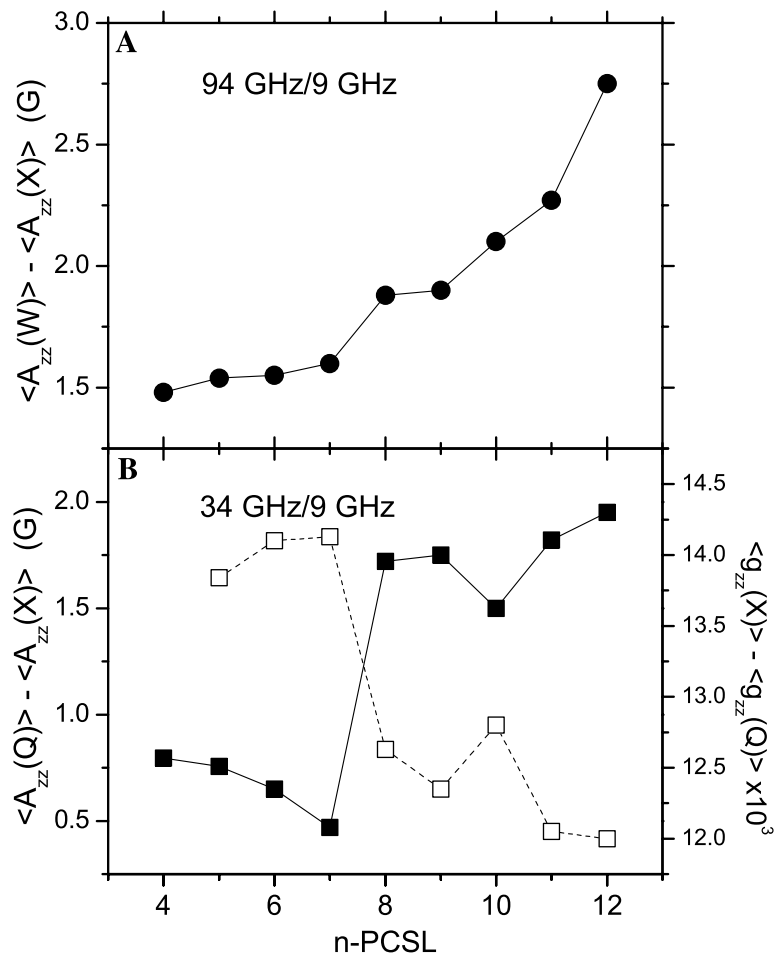

Fig. 3. Dependence of the experimental difference in effective partially averaged hyperfine splittings: (A) $\Delta A_{z z}^{w x}=\left\langle A_{z z}(W)\right\rangle-\left\langle A_{z z}(X)\right\rangle$, and (B) $\Delta A_{z z}^{q x}=\left\langle A_{z z}(Q)\right\rangle-\left\langle A_{z z}(X)\right\rangle$, on the spin-label position, $n$, in the phospholipid chain. Values for the difference in partially averaged effective $g_{z z^{-}}$ values, $\Delta g_{z z}^{q x}=\left\langle g_{z z}(X)\right\rangle-\left\langle g_{z z}(Q)\right\rangle$, are also given in (B) (right-hand ordinate). Data are obtained from the $94-\mathrm{GHz}(W), 34-\mathrm{GHz}(Q)$ and 9$\mathrm{GHz}(X)$ EPR spectra of the $n$-PCSL spin labels in membranes of DMPC $+40 \mathrm{~mol} \%$ cholesterol at $30^{\circ} \mathrm{C}$.

spectra, an effective partially averaged $\left\langle g_{z z}\left(m_{I}\right)\right\rangle$ parameter was calculated from the field position $\left\langle H_{z z}\left(m_{I}\right)\right\rangle$ at the turning point corresponding to the $z$-canonical orientation for the $m_{I}=-1$ hyperfine line (i.e., the high-field peak). As for the partially averaged hyperfine splitting, the difference in effective partially averaged $g_{z z}$-parameters, $\Delta g_{z z}^{q x}=\left\langle g_{z z}(X)\right\rangle-\left\langle g_{z z}(Q)\right\rangle$, is used as a dynamically sensitive quantity. Fig. 4 gives the dependence of $\Delta g_{z z}^{q x}$ on rotational diffusion coefficient $\left(D_{R \perp}\right)$ for different values of the order parameter $\left(S_{z z}\right)$. Experimental values of the $\left\langle g_{z z}\left(m_{I}\right)\right\rangle$ parameter at 9 and $34 \mathrm{GHz}$ were determined from the relation: $\left\langle g_{z z}\left(m_{I}\right)\right\rangle=h v / \beta\left\langle H_{z z}\left(m_{I}\right)\right\rangle$. These effective partially averaged $g_{z z}$-parameters are given in Fig. 3B, for the different $n$-PCSL in membranes of DMPC $+40 \mathrm{~mol} \%$ cholesterol at $30^{\circ} \mathrm{C}$.

By using Fig. 4, it is found that the experimental values of $\Delta g_{z z}^{q x}$ for 5-, 6-, and 7-PCSL correspond to diffusion coefficients $D_{R \perp}$ and order parameters $S_{z z}$ in the range $7.9 \times 10^{6} \mathrm{~s}^{-1}$ and 0.6 to $1.6 \times 10^{7} \mathrm{~s}^{-1}$ and 0.85 , which are closer to the values obtained from spectral simulations (cf. Table 1). However, there are still distinct differences between the values deduced from $\Delta g_{z z}^{q x}$ and those obtained from spectral simulations. These discrepancies between calibrations and simulations can be resolved by a hybrid

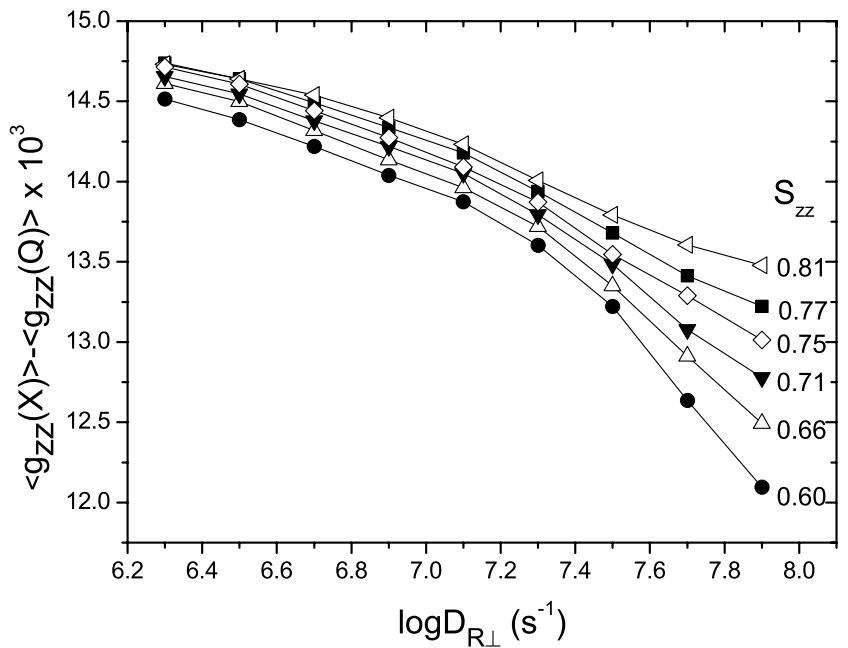

Fig. 4. Dependences of the simulated difference in effective partially averaged $g_{z z}$-values at $9 \mathrm{GHz} \quad(X)$ and $34 \mathrm{GHz} \quad(Q)$, $\Delta g_{z z}^{q x}=\left\langle g_{z z}(X)\right\rangle-\left\langle g_{z z}(Q)\right\rangle$, on rotational diffusion coefficient $\left(D_{R \perp}\right)$ for different values of the order parameter $\left(S_{z z}\right)$. Spectral simulations are performed with the stochastic Liouville equation.

approach that is given in a later section. First, we examine a different membrane system for which the slow-motional components are more pronounced.

\subsection{Multifrequency simulations for DMPC $+5 \mathrm{~mol} \%$ cholesterol}

EPR spectra of several $n$-PCSL $(n=5,7,9)$ in DMPC membranes containing $5 \mathrm{~mol} \%$ cholesterol were studied in the gel phase $\left(\mathrm{L}_{\beta}\right)$ at $10{ }^{\circ} \mathrm{C}$. As for DMPC membranes with $40 \mathrm{~mol} \%$ cholesterol, the tensor components averaged over fast motion were determined from $94-\mathrm{GHz}$ simulations and the dynamic parameters of slow diffusional motion were determined from $9-\mathrm{GHz}$ simulations. The values of $D_{R \perp}$, $D_{R / /}$, and $S_{z z}$ obtained from the latter are included in Table 2. Simulations of the $34-\mathrm{GHz}$ spectra were performed first with fixed input magnetic and dynamic parameters taken

Table 2

Dynamic parameters, $D_{R \perp}(Q), D_{R / /}(Q)$, and $S_{z z}(Q)$, characterising slow overall motion of $n$-PCSL spin labels in membranes of DMPC $+5 \mathrm{~mol} \%$ cholesterol at $10{ }^{\circ} \mathrm{C}$, obtained from simulations of 34-GHz EPR spectra ${ }^{a}$

\begin{tabular}{llllllll}
\hline$n$ & $S_{z z}(X)$ & $S_{z z}(Q)$ & $\begin{array}{l}D_{R \perp}(X) \\
\left(\mathrm{s}^{-1}\right)\end{array}$ & $\begin{array}{l}D_{R \perp}(Q) \\
\left(\mathrm{s}^{-1}\right)\end{array}$ & $\begin{array}{l}D_{R / /}(X) \\
\left(\mathrm{s}^{-1}\right)\end{array}$ & $\begin{array}{l}D_{R / /}(Q) \\
\left(\mathrm{s}^{-1}\right)\end{array}$ & $R \chi^{2}$ \\
\hline 5 & 0.85 & 0.85 & $2.8 \times 10^{7}$ & $4.5 \times 10^{6}$ & $1.0 \times 10^{7}$ & $3.5 \times 10^{6}$ & 82 \\
& & & & & & & \\
7 & 0.76 & 0.85 & $5.8 \times 10^{7}$ & $4.0 \times 10^{6}$ & $1.7 \times 10^{7}$ & $2.5 \times 10^{6}$ & 17.2 \\
& & 0.84 & & $2.0 \times 10^{6}$ & & $2.9 \times 10^{6}$ & 17.0 \\
& 0.83 & & $2.0 \times 10^{6}$ & & $2.8 \times 10^{6}$ & 17.0 \\
& & 0.78 & & $1.2 \times 10^{6}$ & & $2.3 \times 10^{6}$ & 17.0 \\
& \multirow{3}{*}{0.66} & 0.75 & $1.4 \times 10^{7}$ & $4.4 \times 10^{5}$ & $3.7 \times 10^{7}$ & $5.2 \times 10^{6}$ & 98 \\
& & & & $4.5 \times 10^{5}$ & & $5.5 \times 10^{6}$ & 98 \\
& & 0.78 & & $5.5 \times 10^{5}$ & & $5.6 \times 10^{6}$ & 97 \\
& 0.82 & & $5.4 \times 10^{5}$ & & $5.8 \times 10^{6}$ & 97 \\
\hline
\end{tabular}

${ }^{a}$ Corresponding initial parameters obtained from simulation of $9-\mathrm{GHz}$ spectra are given by $D_{R \perp}(X), D_{R / /}(X)$, and $S_{z z}(X)$. 
from the 94- and 9-GHz simulations, respectively. The fits to the experimental $34-\mathrm{GHz}$ spectra were unsatisfactory for all $n$-PCSL spin-label positions $\left(R \chi^{2} \geqslant 200\right)$. Subsequent variation of the dynamic parameters results in a substantial improvement of the fit and produces lower values of $D_{R \perp}$ and $D_{R / /}$, relative to those obtained from $9-\mathrm{GHz}$ simulations (see Fig. 5 and Table 2). It will be noted that, compared with the $n$-PCSL spectra in the liquid-ordered phase (Fig. 1), the spectra in Fig. 5 evidence considerable spin-spin broadening. This is a characteristic feature of these spin probes, particularly those such as 9-PCSL that are labelled towards the middle of chain, when they are intercalated in gel-phase lipid bilayers (see [14]). This is allowed for in the simulations by increasing the linewidths, but it somewhat limits the sensitivity of the simulations to the dynamic parameters.

Again, determination of the diffusion coefficient for axial rotation from the $34-\mathrm{GHz}$ simulations is superior to that from the 9-GHz simulations, because the low-field $x$ - and $y$-turning points are resolved in the $34-\mathrm{GHz}$ spectra (see Fig. 5), but not in the 9-GHz spectra. (It should be noted, however, that in some cases several local minima result in different sets of dynamic parameters with very similar values of $R \chi^{2}$, see, for example, Table 2).

As for membranes of DMPC $+40 \mathrm{~mol} \%$ cholesterol, values of $\Delta A_{z z}^{q x}$ and $\Delta A_{z z}^{w x}$ were also determined from the 9-, 34-, and 94-GHz EPR spectra of $n$-PCSL in membranes of DMPC $+5 \mathrm{~mol} \%$ cholesterol in the gel phase at $10{ }^{\circ} \mathrm{C}$.

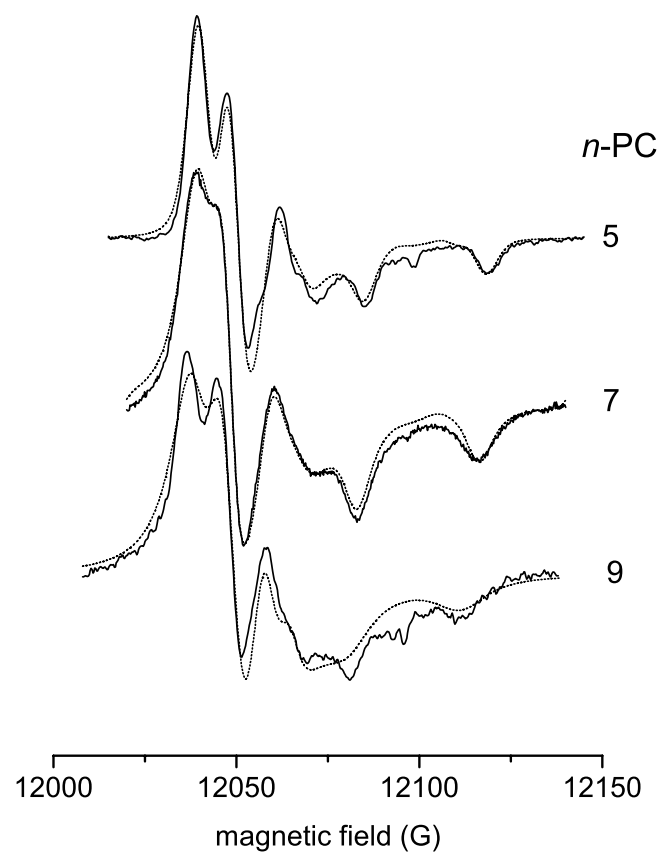

Fig. 5. Experimental (solid lines) and simulated (dotted lines) $34-\mathrm{GHz}$ EPR spectra of $n$-PCSL spin labels in membranes of DMPC $+5 \mathrm{~mol} \%$ cholesterol at $10{ }^{\circ} \mathrm{C}$. The position, $n$, of chain spin-labelling is indicated on the figure. Partially averaged spin-Hamiltonian tensors were obtained from simulation of corresponding $94-\mathrm{GHz}$ spectra. Dynamic parameters used in the simulations are given in Table 2.
These values, which are given in Fig. 6, are greater than for the membranes of DMPC $+40 \mathrm{~mol} \%$ cholesterol in the liquid-ordered phase at $30^{\circ} \mathrm{C}$. As deduced from the calibrations in Fig. 2, the multifrequency $\Delta A_{z z}^{q x}$ and $\Delta A_{z z}^{w x}$ parameters correspond to values of $D_{R \perp}$ that are significantly higher than those given in Table 2 which are obtained from direct simulation of the $34-\mathrm{GHz}$ spectra. This is a feature found already in the analysis of the $n$ PCSL spectra from membranes of DMPC $+40 \mathrm{~mol} \%$ cholesterol at $30^{\circ} \mathrm{C}$.

Evidently, the fast motion is not sufficiently rapid at $34 \mathrm{GHz}$ to give rise to complete motional averaging that results in the effective Hamiltonian $\langle\mathscr{H}(\Omega)\rangle$. Rotational diffusion coefficients for the fast motional component appear to be on the order of $10^{9} \mathrm{~s}^{-1}$, according to simulations of the high-field spectra from 16-PCSL at $250 \mathrm{GHz}$ [2]. Furthermore, the spectra of the $n$-PCSL at $94 \mathrm{GHz}$ are not entirely free from slow-motion contributions for chain positions $n>7$ [3]. Significantly, it is for just these label positions that fits to the $34-\mathrm{GHz}$ spectra deteriorate when using partially averaged tensor values obtained from the 94- $\mathrm{GHz}$ simulations (see Fig. 1). These effects may be accounted for, in a semiquantitative manner, by adjusting the $A_{i i}$ and $g_{i i}$ tensor components for the $34-\mathrm{GHz}$ (and 94-GHz) simulations when constructing the calibrations. In this case, one obtains higher values of $\Delta A_{z z}^{w x}$ and $\Delta A_{z z}^{q x}$ in calibration plots of the type given in Fig. 2. Moreover, using these same, less averaged $A_{i i}$ and $g_{i i}$ components in spectral simulations yields higher values of $D_{R \perp}$ and $S_{z z}$ that are closer to those obtained from the multifrequency calibrations. This strategy is used for final refinement of the spectral simulations in the next section.

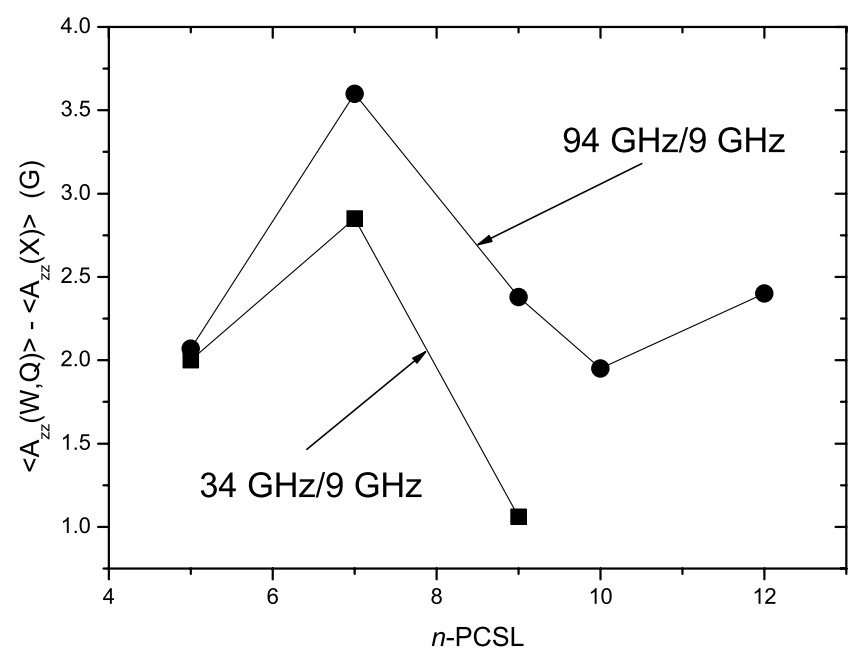

Fig. 6. Dependence of the experimental difference in effective partially averaged hyperfine splittings: $\Delta A_{z z}^{w x}=\left\langle A_{z z}(W)\right\rangle-\left\langle A_{z z}(X)\right\rangle$ (circles), and $\Delta A_{z z}^{q x}=\left\langle A_{z z}(Q)\right\rangle-\left\langle A_{z z}(X)\right\rangle$ (squares), on the spin-label position, $n$, in the phospholipid chain. Data are obtained from the $94-\mathrm{GHz}(W), 34-\mathrm{GHz}$ $(Q)$, and $9-\mathrm{GHz}(X)$ EPR spectra of the $n$-PCSL spin labels in membranes of DMPC $+5 \mathrm{~mol} \%$ cholesterol at $10{ }^{\circ} \mathrm{C}$. 


\subsection{Refinement of multifrequency simulations}

For each $n$-PCSL in DMPC $+40 \mathrm{~mol} \%$ cholesterol at $30{ }^{\circ} \mathrm{C}$, the input values of $A_{z z}$ for the $34-\mathrm{GHz}$ simulations were increased to such an extent that the resulting values of $\Delta A_{z z}^{q x}$ were equal to the experimental ones, at the values of $D_{R \perp}$ and $S_{z z}$ that are given in Table 1. Specifically, the input values of $A_{z z}$ were increased by $0.4-1.3 \mathrm{G}$, depending on spin-label position, $n$. For the $g$-tensor components, values intermediate between the averaged and rigid (polaritycorrected) ones were taken. These values were varied in the simulations and are summarised in Table 3 . The resulting spectral simulations that give the best fit are shown in Fig. 7, and the corresponding values of $D_{R \perp}, D_{R / /}$, and $S_{z z}$ are presented in Table 3.

Table 3

Spin Hamiltonian tensor components and dynamic parameters $D_{R \perp}, D_{R / /}$, and $S_{z z}$ determined from simulations of $34 \mathrm{GHz}$ EPR spectra from $n$ PCSL spin labels in membranes of DMPC $+40 \mathrm{~mol} \%$ cholesterol at $30{ }^{\circ} \mathrm{C}$

\begin{tabular}{|c|c|c|c|c|c|c|}
\hline$n$ & $\begin{array}{l}\left(g_{i i}-2\right) \times 10^{4} \\
(x, y, z)\end{array}$ & $\begin{array}{l}A_{z z} \\
(\mathrm{G})^{\mathrm{a}}\end{array}$ & $\begin{array}{l}D_{R \perp} \\
\left(\mathrm{s}^{-1}\right) \\
\end{array}$ & $\begin{array}{l}D_{R / /} \\
\left(\mathrm{s}^{-1}\right) \\
\end{array}$ & $S_{z z}$ & $R \chi^{2}$ \\
\hline 4 & $\begin{array}{l}83.9 \\
57.3 \\
21.2\end{array}$ & 33.8 & $6.3 \times 10^{6}$ & $7.9 \times 10^{6}$ & $0.85(7.3)^{\mathrm{b}}$ & 6 \\
\hline 5 & $\begin{array}{l}84.0 \\
57.6 \\
21.3\end{array}$ & 33.6 & $7.4 \times 10^{6}$ & $1.0 \times 10^{7}$ & $0.83(6.3)^{\mathrm{b}}$ & 114 \\
\hline 6 & $\begin{array}{l}84.1 \\
57.6 \\
21.6\end{array}$ & 33.5 & $5.6 \times 10^{6}$ & $7.4 \times 10^{6}$ & $0.80(5.6)^{\mathrm{b}}$ & 70 \\
\hline 7 & $\begin{array}{l}83.9 \\
57.6 \\
22.3\end{array}$ & 33.1 & $5.4 \times 10^{6}$ & $1.3 \times 10^{7}$ & $0.82(6.2)^{\mathrm{b}}$ & 38 \\
\hline 8 & $\begin{array}{l}84.4 \\
57.6 \\
22.8\end{array}$ & 32.9 & $1.1 \times 10^{7}$ & $1.3 \times 10^{7}$ & $0.75(4.5)^{\mathrm{b}}$ & 292 \\
\hline 9 & $\begin{array}{l}87.3 \\
59.1 \\
22.2\end{array}$ & 32.1 & $1.3 \times 10^{7}$ & $2.0 \times 10^{7}$ & $0.61(3.05)^{b}$ & 410 \\
\hline 10 & $\begin{array}{l}83.0 \\
58.8 \\
23.2\end{array}$ & 30.9 & $2.4 \times 10^{7}$ & $1.6 \times 10^{7}$ & $0.66(3.45)^{\mathrm{b}}$ & 52 \\
\hline 11 & $\begin{array}{l}82.4 \\
59.6 \\
23.8\end{array}$ & 31.0 & $2.7 \times 10^{7}$ & $1.5 \times 10^{7}$ & $0.66(3.45)^{\mathrm{b}}$ & 52 \\
\hline 12 & $\begin{array}{l}82.4 \\
59.6 \\
23.0\end{array}$ & 31.3 & $2.5 \times 10^{7}$ & $1.8 \times 10^{7}$ & $0.66(3.45)^{b}$ & 49 \\
\hline
\end{tabular}

The tensor components were chosen to provide agreement with experimental determinations of $\Delta A_{z z}^{q x}$.

${ }^{\text {a }}$ Values of $A_{x x}$ and $A_{y y}$ were in the range $5.0-5.5 \mathrm{G}$, and variations within these limits practically did not influence the simulated lineshapes.

${ }^{\mathrm{b}}$ Values in parentheses are the strengths of the orientational pseudopotential $-\varepsilon_{20} / k_{\mathrm{B}} T$ (with $\varepsilon_{22}=0$ in) Eq. (1) that correspond to the $S_{z z}$ order parameters listed.

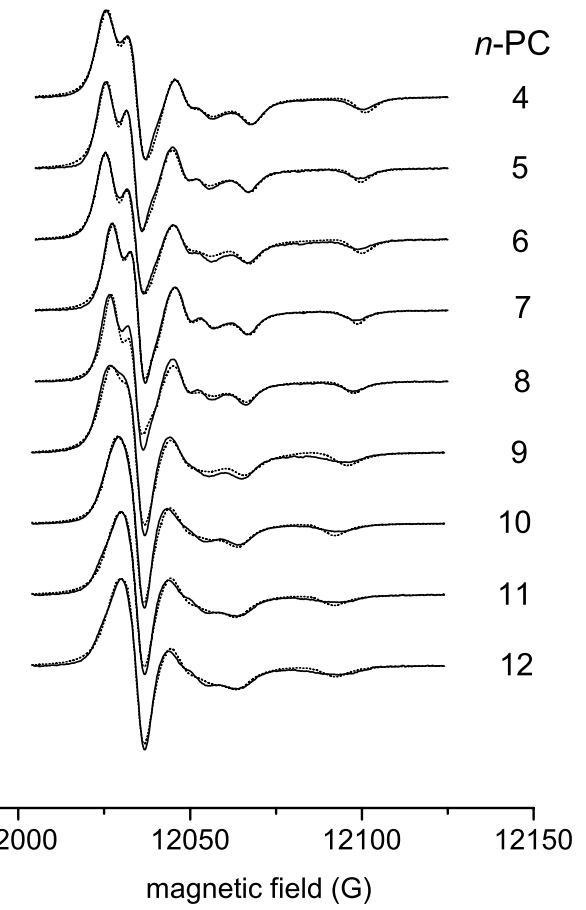

Fig. 7. Experimental (solid lines) and simulated (dotted lines) $34-\mathrm{GHz}$ EPR spectra of $n$-PCSL spin labels in membranes of DMPC $+40 \mathrm{~mol} \%$ cholesterol at $30^{\circ} \mathrm{C}$. The position, $n$, of chain spin-labelling is indicated on the figure. The input spin-Hamiltonian tensors were adjusted to achieve agreement between experimental and simulated values of $\Delta A_{z z}^{q x}$. Simulation parameters are given in Table 3.

As already mentioned, because of the relative insensitivity of the least squares algorithm to the less intense of the first-derivative spectral features, an increase in the input value of $A_{z z}$ does not result in a noticeable change in the values of $R \chi^{2}$. The optimum input values of the $g_{x x}$ and $g_{y y}$ components are higher by $0.2-2 \times 10^{-4}$, and of the $g_{z z}$ components are lower by $0.2-2 \times 10^{-4}$ (see Table 3 ), than the partially averaged $g_{i i}$ components that were used originally for the 9-GHz simulations [3].

The 9-GHz EPR spectra of the $n$-PCSL spin labels in $\mathrm{DMPC}+40 \mathrm{~mol} \%$ cholesterol at $30^{\circ} \mathrm{C}$ were therefore resimulated by using the revised spin-Hamiltonian parameters that were established from the $34-\mathrm{GHz}$ simulations. Results of these 9-GHz simulations are shown in Fig. 8, and the corresponding simulation parameters are listed in Table 4. Satisfactory simulations of the $9-\mathrm{GHz}$ spectra are achieved without further adjustment, or with only very minor adjustment, of the revised spin-Hamiltonian parameters. As seen from comparison of Tables 3 and 4, the values of $D_{R \perp}$ and $S_{z z}$ that are obtained for the slow off-axial motion become rather similar from both $34-$ and $9-\mathrm{GHz}$ simulations, when using the revised spin-Hamiltonian parameters. The values of $D_{R / /}$ for the slow axial rotation are also in reasonable agreement, although, as already noted, the axial diffusion parameters are determined far more sensitively from the $34-\mathrm{GHz}$ spectra.

At least part of the reason that the strategy of readjusting the spin-Hamiltonian tensors is effective, therefore 


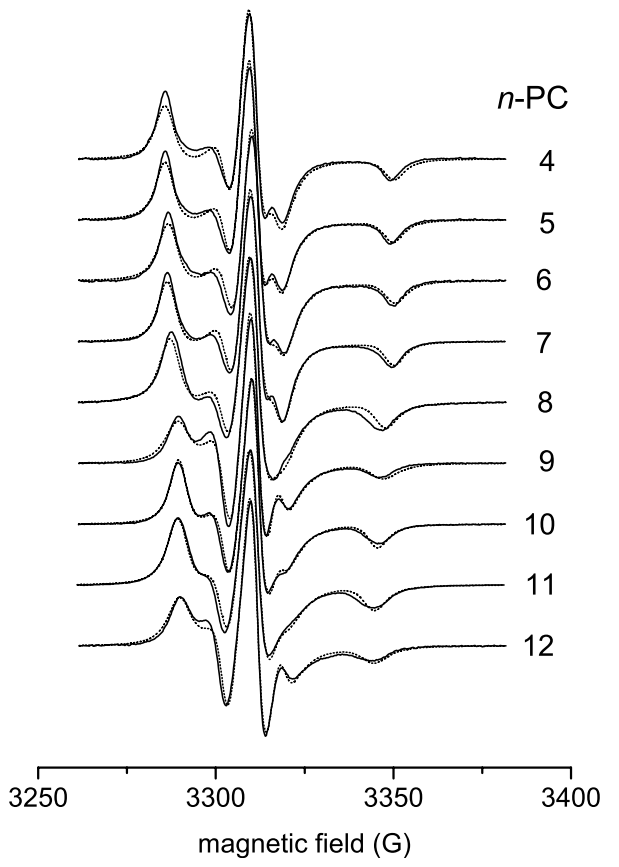

Fig. 8. Experimental (solid lines) and simulated (dotted lines) 9-GHz EPR spectra of $n$-PCSL spin labels in membranes of DMPC $+40 \mathrm{~mol} \%$ cholesterol at $30^{\circ} \mathrm{C}$. The position, $n$, of chain spin-labelling is indicated on the figure. The input spin-Hamiltonian tensors are the same or close to those used for simulations of the 34-GHz spectra in Fig. 7. Simulation parameters are given in Table 4.

might be that it allows for non-axial contributions to the slow motion (in addition to axial contributions). These could be close to the rigid limit at $94 \mathrm{GHz}$, and would not contribute to the $9-\mathrm{GHz}$ spectra because these are sensitive only to axial rotation. The method used for simulation of the 9- and $34-\mathrm{GHz}$ spectra cannot be applied to the $94-\mathrm{GHz}$ spectra, because the slow-motion simulation programmes do not incorporate non-axial rotation of limited azimuthal amplitude. This non-axiality is a dominant feature in the 94-GHz spectra [9], but will be considerably attenuated at $34 \mathrm{GHz}$.

\section{Conclusions}

Inclusion of spectra at an intermediate microwave frequency $(34 \mathrm{GHz})$, between that of the high $(94 \mathrm{GHz})$ and low $(9 \mathrm{GHz})$ frequency regimes, greatly improves the precision in determination of the dynamic parameters associated with the slow components of the multi-component rotational diffusion exhibited by spin-labelled lipids in membranes. This is especially the case for the slow axial diffusion.

To simulate the spectra at the intermediate microwave frequency, partially averaged spin-Hamiltonian tensors are taken from fast-motional simulations of the $94-\mathrm{GHz}$ spectra and are used in slow-motional simulations based on the stochastic Liouville equation. The slow-motion parameters obtained in this way differ, however, from those obtained by simulating the $9-\mathrm{GHz}$ spectra with the same procedure,
Table 4

Dynamic parameters $D_{R \perp}, D_{R / /}$, and $S_{z z}$ determined from simulations of 9-GHz EPR spectra from $n$-PCSL spin labels in membranes of DMPC $+40 \mathrm{~mol} \%$ cholesterol at $30^{\circ} \mathrm{C}$

\begin{tabular}{|c|c|c|c|c|c|c|}
\hline$n$ & $\begin{array}{l}\left(g_{i i}-2\right) \times 10^{4} \\
(x, y, z)\end{array}$ & $\begin{array}{l}A_{z z} \\
(\mathrm{G})^{\mathrm{a}}\end{array}$ & $\begin{array}{l}D_{R \perp} \\
\left(\mathrm{s}^{-1}\right)\end{array}$ & $\begin{array}{l}D_{R / /} \\
\left(\mathrm{s}^{-1}\right)\end{array}$ & $S_{z z}$ & $R \chi^{2}$ \\
\hline 4 & $\begin{array}{l}83.8 \\
57.4 \\
21.3\end{array}$ & 33.8 & $1.0 \times 10^{7}$ & $9.1 \times 10^{6}$ & $0.845(7.0)^{\mathrm{b}}$ & 215 \\
\hline 4 & $\begin{array}{l}83.9 \\
57.3 \\
21.2\end{array}$ & 33.8 & $7.9 \times 10^{6}$ & $1.0 \times 10^{7}$ & $0.835(6.5)^{\mathrm{b}}$ & 229 \\
\hline 5 & $\begin{array}{l}84.0 \\
57.6 \\
21.3\end{array}$ & 33.6 & $7.9 \times 10^{6}$ & $1.3 \times 10^{7}$ & $0.83(6.3)^{b}$ & 117 \\
\hline 6 & $\begin{array}{l}84.1 \\
57.6 \\
21.6\end{array}$ & 33.4 & $7.1 \times 10^{6}$ & $1.0 \times 10^{7}$ & $0.80(5.6)^{\mathrm{b}}$ & 48 \\
\hline 7 & $\begin{array}{l}83.9 \\
57.6 \\
22.3\end{array}$ & 33.1 & $5.6 \times 10^{6}$ & $1.3 \times 10^{7}$ & $0.825(6.2)^{\mathrm{b}}$ & 609 \\
\hline 8 & $\begin{array}{l}84.4 \\
57.6 \\
22.8\end{array}$ & 32.7 & $1.4 \times 10^{7}$ & $1.0 \times 10^{7}$ & $0.75(4.5)^{\mathrm{b}}$ & 399 \\
\hline 9 & $\begin{array}{l}87.3 \\
59.1 \\
22.2\end{array}$ & 31.9 & $1.4 \times 10^{7}$ & $1.1 \times 10^{7}$ & $0.61(3.05)^{\mathrm{b}}$ & 681 \\
\hline 10 & $\begin{array}{l}83.0 \\
58.8 \\
23.2\end{array}$ & 30.9 & $1.9 \times 10^{7}$ & $2.0 \times 10^{7}$ & $0.66(3.45)^{\mathrm{b}}$ & 9 \\
\hline 11 & $\begin{array}{l}82.4 \\
59.6 \\
23.8\end{array}$ & 31.0 & $2.5 \times 10^{7}$ & $1.4 \times 10^{7}$ & $0.66(3.45)^{\mathrm{b}}$ & \\
\hline 12 & $\begin{array}{l}82.4 \\
59.6 \\
23.0\end{array}$ & 30.9 & $2.5 \times 10^{7}$ & $1.8 \times 10^{7}$ & $0.66(3.45)^{\mathrm{b}}$ & 154 \\
\hline
\end{tabular}

The spin-Hamiltonian tensor components used were the same or close to those used for the 34-GHz simulations.

${ }^{a}$ Values of $A_{x x}$ and $A_{y y}$ were in the range $5.0-5.5 \mathrm{G}$, and variations within these limits practically did not influence the simulated lineshapes.

${ }^{\mathrm{b}}$ Values in parentheses are the strengths of the orientational pseudopotential $-\varepsilon_{20} / k_{\mathrm{B}} T$ (with $\varepsilon_{22}=0$ in) Eq. (1) that correspond to the $S_{z z}$ order parameters listed.

possibly because of non-axial contributions to the slow motion to which 9-GHz spectra are insensitive. Agreement between the dynamic parameters obtained from 9- and 34- $\mathrm{GHz}$ simulations can be achieved by adjustment of the effective degree of rapid motional averaging of the spinHamiltonian tensors. Calibrations of the multifrequency parameters $\Delta A_{z z}^{q x}$ and $\Delta g_{z z}^{q x}$ by simulations are used to guide this optimization step. In this way, it is possible to obtain a consistent description of the slow axial motion of the lipid chains in terms of the rotational diffusion coefficients $D_{R \perp}$ and $D_{R / /}$, and the long-axis order parameter $S_{z z}$. 


\section{Acknowledgments}

We thank Frau B. Angerstein for synthesis of spin-labelled lipids. This work was supported by the Schwerpunktprogramm SPP 1051 "High-field EPR in Biology, Chemistry and Physics" of the Deutsche Forschungsgemeinschaft.

\section{References}

[1] M. Moser, D. Marsh, P. Meier, K.-H. Wassmer, G. Kothe, Chain configuration and flexibility gradient in phospholipid membranes. Comparison between spin-label electron spin resonance and deuteron nuclear magnetic resonance, and identification of new conformations, Biophys. J. 55 (1989) 111-123.

[2] Y. Lou, M. Ge, J.H. Freed, A multifrequency ESR study of the complex dynamics of membranes, J. Phys. Chem. B 105 (2001) 11053 11056.

[3] V.A. Livshits, D. Kurad, D. Marsh, Simulation studies on high-field EPR of lipid spin labels in cholesterol-containing membranes, J. Phys. Chem. B 108 (2004) 9403-9411.

[4] V.A. Livshits, D. Marsh, High-field EPR spectra of spin labels in membranes, in: O.Y. Grinberg, L.J. Berliner (Eds.), Very High Frequency (VHF) ESR/EPR, Kluwer Academic, New York, 2004, pp. 431-464.

[5] J.S. Hyde, K.V.S. Rao, Determination of the rotational correlation time and the rigid limit hyperfine coupling from the dependence of spin-label EPR spectra on microwave frequency, J. Magn. Reson. 38 (1980) 313-317.
[6] J.H. Freed, Theory of slowly tumbling ESR spectra for nitroxides, in: L.J. Berliner (Ed.), Spin Labeling, Theory and Applications, Academic Press, New York, 1976, pp. 53-132.

[7] W.L. Hubbell, H.M. McConnell, Molecular motion in spin-labelled phospholipids and membranes, J. Am. Chem. Soc. 93 (1971) 314-326.

[8] D. Marsh, A. Watts, Spin-labeling and lipid-protein interactions in membranes, in: P.C. Jost, O.H. Griffith (Eds.), Lipid-Protein Interactions, vol. 2, Wiley-Interscience, New York, 1982, pp. 53-126.

[9] D. Kurad, G. Jeschke, D. Marsh, Lateral ordering of lipid chains in cholesterol-containing membranes: high-field spin-label EPR, Biophys. J. 86 (2004) 264-271.

[10] R. Cassol, M.T. Ge, A. Ferrarini, J.H. Freed, Chain dynamics and the simulation of electron spin resonance spectra from oriented phospholipid membranes, J. Phys. Chem. B 101 (1997) 8782-8789.

[11] A. Polimeno, J.H. Freed, Slow motional ESR in complex fluids-the slowly relaxing local structure model of solvent cage effects, J. Phys. Chem. 99 (1995) 10995-11006.

[12] D.J. Schneider, J.H. Freed, Calculating slow motional magnetic resonance spectra: a user's guide, in: L.J. Berliner, J. Reuben (Eds.), Spin-Labeling. Theory and Applications., Plenum Publishing, New York, 1989, pp. 1-76.

[13] D.E. Budil, S. Lee, S. Saxena, J.H. Freed, Nonlinear least-squares analysis of slow-motion EPR spectra in one and two dimensions using a modified Levenberg-Marquardt algorithm, J. Magn. Reson. A 120 (1996) 155-189.

[14] P. Fajer, A. Watts, D. Marsh, Saturation transfer, continuous wave saturation, and saturation recovery electron spin resonance studies of chain-spin labeled phosphatidylcholines in the low temperature phases of dipalmitoyl phosphatidylcholine bilayers. Effects of rotational dynamics and spin-spin interactions, Biophys. J. 61 (1992) 879-891. 